\title{
Distributed Personal Learning Environments: Towards a Suitable Architecture
}

\author{
http://dx.doi.org/10.3991/ijet.v8iS2.2764 \\ Mario Manso Vázquez, Martín Llamas Nistal \\ University of Vigo, Vigo, Spain
}

\begin{abstract}
Personal Learning Environment is a reference to future learning. In this paper we present a multi-level analysis of the options and requirements in the universe of PLE, analyzing the content management system as the main tool of the learning environment. There are two principal models: distributed applications and mashups. Both have advantages and disadvantages, and they also share a big issue: lack of inter-application communication. We are trying to find a solution to this problem presenting an architecture for distributed applications model, so that transparency to the uses will be maintained.
\end{abstract}

Index Terms-Personal Learning Environment; SelfRegulated Learning; information management; social networking; inter-application communication; distributed applications.

\section{INTRODUCTION}

During the last decade, technologies have been introduced in education mainly to extend the reach of the classroom. The use of LMS (Learning Management Systems) and VLEs (Virtual Learning Environments) has allowed us to complement classroom teaching, as well as to teach full online courses [1]. But Van Harmelen [2] pointed out back in 2006 that VLEs and LMSs don't deal as well as they could with the needs of learners. At the same time, online search tools have replaced in many cases the use of reference books and visiting the library. There is a huge amount of information available on the internet, that can be unmanageable [3].

Personal Learning Environments (PLEs) were created as a concept designed to meet the needs of Self-Regulated Learning (SRL), initially informal and non institutional. SRL refers to student's control over their learning process, i.e. controlling their cognition as well as resources for learning. This meta-cognitive element is an important part of self-regulated learning, i.e. awareness of one's cognition and self-regulation of one's cognition [4]. Advances in new technologies, the amount of information available on the Internet and the evolution of Web 2.0 has enabled the birth of these environments in which the student self-organize his/her learning and choose from the applications to use to his/her learning goals, all this while working in a social and collaborative way with others, whether students, teachers, enthusiast of a subject, etc. PLEs are based on a different pedagogical approach [5], leading to a change in the methodology that makes knowledge to be consolidated in a more stable way and allow the learners to develop their metacognitive skills. On the other side, even though students may be familiar with the Internet and social software, they may not necessarily know how to use the Internet and social software for learning, probably because they have never had the opportunity to do so in formal educational situations [6]. The scientific community wants to prove that these personal environments are a reference to future learning, and many experiments to test the validity of the concept are being carried out [6][7][8].

After years of debate about how PLEs should be, it seems that both developers and users are finally coming to a common point. The PLE built as a set of distributed applications or as a mashup of web applications are the most successful configurations. This choice is a matter of personal preference, and it is the learner who has to decide which model suits his/her way of working best. We focus on the distributed applications PLE point of view, which is dynamic and customizable. Currently there are many powerful tools to do all the tasks that are needed when using a PLE or any learning environment. It is pointless and a highly difficult technical challenge to create a single tool or environment that offers all that features integrated into a single interface. It seems more practical to choose the best already developed applications and connect them, or to offer an information transfer method or standard which should be compatible with all tools. This is an unresolved problem such as the problem of access to many different tools, not connected among them.

In this paper we analyze the options and requirements of the perfect PLE in section 2. Following this, section 3 is dedicated to the analysis of the two main PLE models, the mashup and the distributed applications PLE. Section 4 deals with information and resource management, analyzing the most interesting options which are social bookmarking, content structures and concept maps. After that we focus on the inter-application communication problem in section 5. With all this information presented, we propose a distributed application PLE solution in section 6. Section 7 is dedicated to related work. In section 8 we put forward some conclusions.

\section{The PERFECT PLE}

Although this is very relative and dependent on personal preferences, we collected some ideas and requirements that a PLE should have from an ideal point of view:

- Student centered (possible tutor support). Student has control over resources, goals and manages his/her own learning.

- Collaborative. A PLE must allow collaboration among teachers, students, experts and any members of the community. 
- Open. It must be open and interoperable, allowing its extension with plug-ins or new applications.

- Customizable. Its construction and functionalities have to be customizable.

- Distributed and infinite content. Students must be able to get contents from multiple sources, and must be able to create more content as well.

- Compatible with standards. The content manager of the ideal PLE should be compliant with the most extended standards.

- Mobile terminal implementation. Some of the features of the PLE could be managed from mobile terminals like smartphones and tablets.

- PLE are not persistent environments. They should evolve according to the learner's objectives and achievements, as well as competence management requirements [1].

- Bridge to educational institutions. A suitable PLE should act as a bridge to educational institutions and formal learning LMSs and VLEs. It would be ideal to have also good connectivity with official content providers.

Some old requirements like the possibility to work offline are not that important today thanks to mobile technology and Internet development. From a functional point of view:

- Knowledge acquisition and discovery: search engine (resources, contacts,...), RSS.

- Knowledge management: bookmarks (resources storing and management), tagging (knowledge classification) and mind maps (knowledge relationships).

- Knowledge exchange: blog, wikis (reflection, knowledge sharing), social annotation (study tool, knowledge sharing) and social tagging (resource classification and discovery).

- Communication: social networks, email, instant messaging, etc.

- Social media and content consumption tools: video, hypermedia, stock photos, music, etc.

- Learning management: planner, calendar, time management, task management, goal management, metacognitive skills developer (through reflection), etc.

\section{MAIN PLE MODELS}

There are several interpretations and concepts about PLE. Ideas are so different that there are publications that specifically deal with this discussion [9]. There are three basic PLE models: all-in-one PLE, mashup PLE and distributed applications PLE. After years of debate about how a PLE should be, it seems that both developers and users are finally coming to a common point. The mashup and distributed applications PLE models are being adopted by the developers due to user preference and practicality.

\section{A. All-In-One PLE}

This is a single tool that offers all the features that a PLE should have into a single interface, all of them being developed specifically to build the tool or included through hard integration. At the same time, this environment should be adaptable and customizable to every user. At the same time, it could be an online or desktop application.

This option has many drawbacks:

- Users won't be able to choose any tool separately; the PLE is a closed package, a single tool with all the functionalities.

- Limited customization capability.

- Limited adaptability.

- There are specific tools to cover each feature with high end quality and functionalities.

- It is a difficult technical challenge to get this done achieving a minimum of adaptability and customization, and offering high end features compared to separated specific tools.

An example of this philosophy is PLEX [10] (see Figure 1).

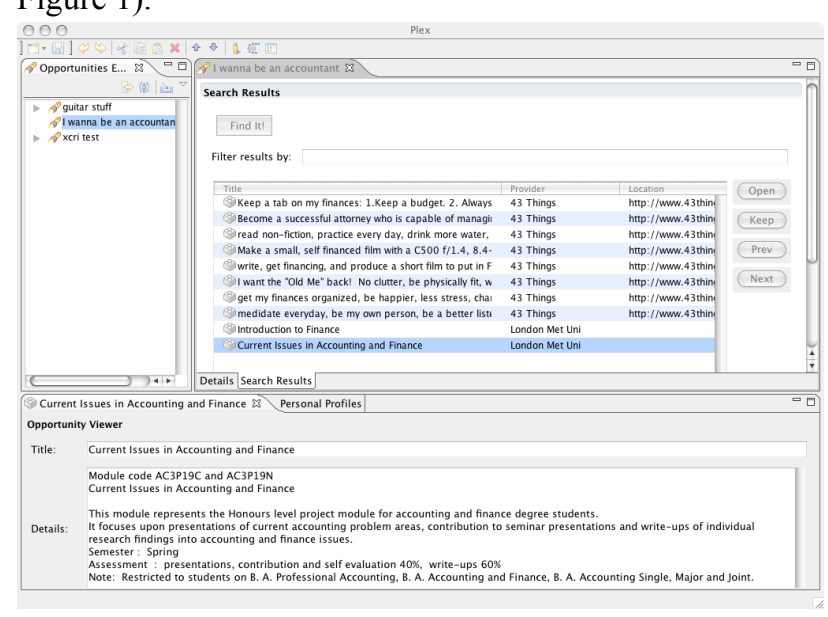

Figure 1. PLEX, a desktop interface PLE [11].

\section{B. Mashup of Applications}

According to Wang, Xiayuan, Chengling and Chunyan [12], a PLE is not only a sum of all used tools, it is a technological realization where social software applications and web services are combined. Considering traditional web experience, users are tied to a single application or they have to manage a set of applications, data sources, etc on their own with limited scaffolding support or lack thereof. The mashup era has emerged in response to the challenge of integrating existing services, data sources and tools to generate new applications [13]. With this in mind, it seems that mashup can be a good choice to build a PLE.

Mashups can include communication apps (email, chat,...), apps to obtain content (search, RSS, ...), manage content (bookmarks, tags, ...), tools to publish and share knowledge (blog, wiki, ...). This may allow collaborative and social learning.

This model has some advantages: 
- Single login. Users don't have to $\log$ in to each application separately.

- Application selection (in some cases). Users are able to choose which application to use for each requirement.

- Screen use. Users can see a lot of information in the same layout, simultaneously.

It also has some disadvantages:

- Isolated applications: applications don't react to other application's changes even if they are related.

- Low screen space per application.

- Limited application features. In some cases, some of the original features of an application could be reduced in a mashup configuration or integration.

- The amount of data available on the screen can be confusing for some users.

- In some cases, the mashup is preconfigured so users are not allowed to choose each application.

A good example of this model is PLEF (Personal Learning Environment Framework) [14], showed in Figure 2. This environment offers a set of Web2.0 services for the users to build a PLE without demanding any integration abilities. It's based on RSS, tags, link lists, gadget integration (SlideShare [15], YouTube [16]...) and users can also upload images or create text. Login can be done through OpenID [17]. It allows some customization, it is social (it can connect to other personal spaces and any element within the PLE can be shared) and it has text or tag searching capabilities [12].

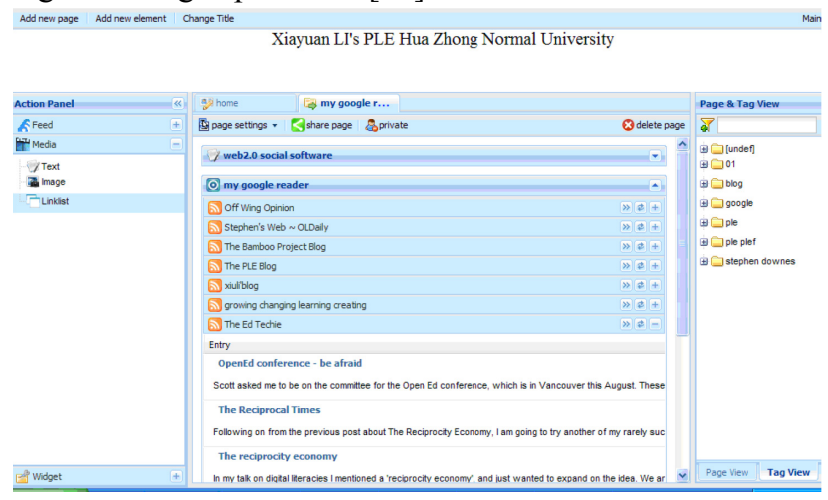

Figure 2. PLEF, a web mashup PLE [12].

At the same time, there are numerous generic tools not developed for learning purposes that are being used as PLEs like Elgg [18], Netvibes [19], Ning [20], Popurls [21], Pageflakes [22],... Some of them like Elgg can be installed to a server to increase privacy level.

\section{Distributed Applications}

According to Adell \& Castañeda [5], a PLE is a collection of tools, resources, connections and activities used by students to learn. This places the student in the center of the action, and the PLE is made of elements that take part in the student's learning, including information sources, methods, references, other students and relationships among them, seeing this from the ICT perspective. This model of PLE is formed by a set of distributed applications that used together for learning becomes a PLE (see Figure 3). Each one of these applications has been created with specific functionalities to cover specific needs, not necessarily related to learning or education. Most of them are generic tools with a social element that allow sharing, discussing, creating,... It is the purpose of the user, combining all these applications in a certain way, what makes them to become a PLE.

This model has some advantages:

- User's freedom. Users can choose all the tools they want to use to build their PLE, with no restrictions.

- Easy updating. It is easy to update each tool separately.

- Optimized interfaces. Each application has its own interface, designed specifically to its purpose, so it is optimized.

- All functionalities. Applications have all their functionalities available at their own interfaces.

It also has some disadvantages:

- Multiple login. Users have to $\log$ in to each application separately and remember multiple login data.

- Multiple URL. Each application has its own URL.

- Multiple interfaces. Users have to learn to use each interface separately.

- Isolated applications: applications don't react to other application's changes even if they are related.

Henri \& Charlier [23] and Sclater [24] affirm that these disadvantages do not make this model robust or scalable for institutional use, but users are the ones with the power to choose which model they prefer. In many cases, they won't change their favorite applications to use a mashup that doesn't integrate them. There are no solutions to this model problems.

\section{INFORMATION AND RESOURCES MANAGEMENT}

Information and learning resources management is one of the keys for this type of learning experience to be successful. There are several options for sorting, searching and accessing the resources. The most interesting options are social bookmarking and tagging, content structures and concept maps. Some of the applications named below have been created specifically to be a PLE itself or to be a part of it. Others have not been conceived with this initial intention in mind, but they offer features that can be essential to manage information efficiently, and are being used massively for learning or work.

\section{A. Social Bookmarking and Tagging}

\section{1) Concept}

This is an alternative with a lot of followers. Tools like Delicious [25] and Diigo [26] offer social tagging based on folksonomies. A Folksonomy [27] is the result of personal free tagging of information and objects (anything with a URL) for one's own retrieval. The use of tags (freeform keywords used to describe digital resources) is done in a social environment (usually shared and open to others). 


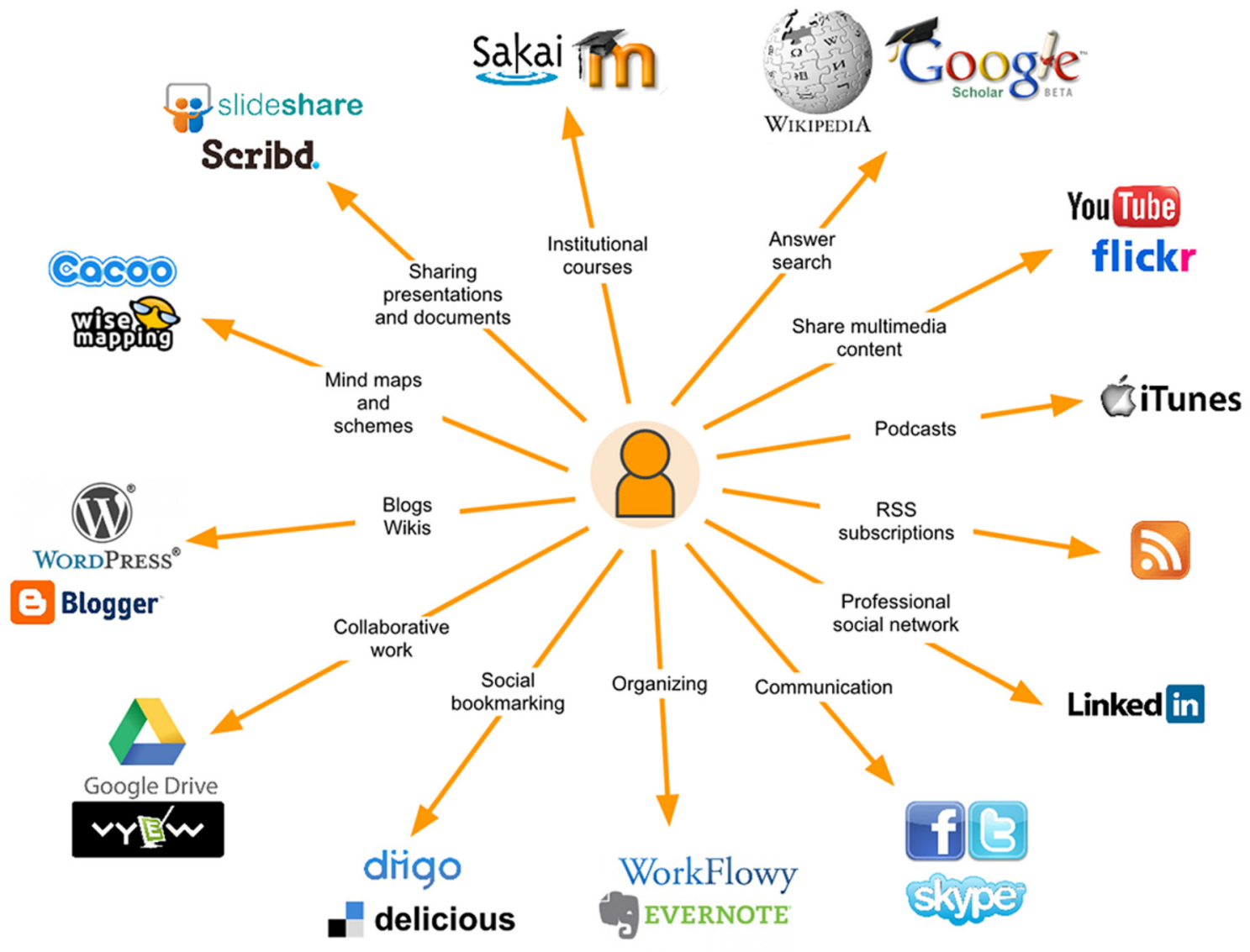

Figure 3. Distributed Application PLE.

Other terms used to refer to a folksonomy are Collaborative Tagging, Social Classification, Social Tagging, or Social Indexation. Collaborative tagging systems in general have some advantages and disadvantages [28]:

- Advantages: Wisdom of crowds, inclusive, current, offer discovery, non-binary, democratic and selfmoderating, follow desire lines, offer insight into user behavior, engender community, offer a low cost alternative and offer usability.

- Disadvantages: No synonym control, problems regarding homonymy and plurals, lack of precision, missing hierarchy, problems with the use of different languages and lack of default visualization.

Collaborative tagging can be a good tool to be integrated into a PLE because it enriches peer interactions and it is a reflective practice which is at the same time supported by other peers. It establishes relations among resources and helps to classify them, allows content discovery and going deeper into any topic.

Diigo deserves a special mention due to some of its features, especially digital highlighting and annotation (see Figure 4). A highlighter and sticky notes or written annotations are tools that every learner uses when studying on paper and their usefulness is undeniable. All these highlights and annotations are also shareable. It is not the only online web highlighter but it is the only social bookmarking tool with these features. On the other hand, it also allows one to create link lists but it does not allow one to create structures.

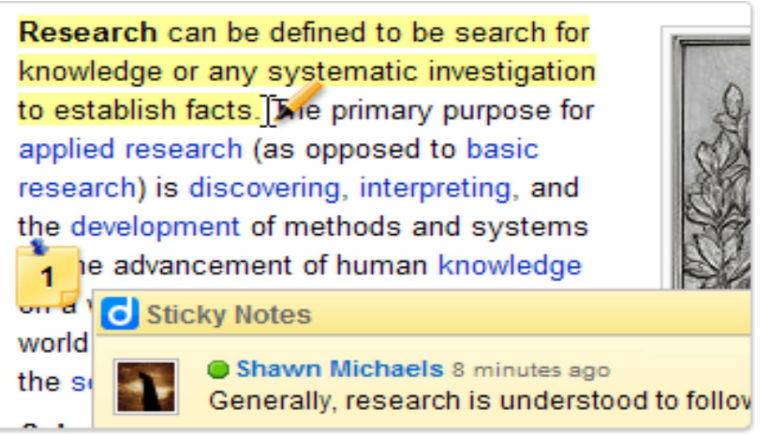

Figure 4. Diigo's web highlighting and annotation.

\section{2) Visualization}

Since folksonomies do not have a natural way of seeing contents, tag-cloud has turned into a common method for visualizing tags. Sánchez \& Llamas [29] made an analysis of tag visualization. Common tag representations are tag clouds, cluster tag clouds (semantically related tags are displayed in clusters, and this in a cloud), elastic tag maps (relationships between tags is represented, navigation based in pivot browsing) and Sixpli (interactive network which employs different imaging methods including elastic nets 3D, 2D and circles, but seems to be currently unavailable). Pivot browsing allows the user to browse the semantic context of a tag, locking it to explore cooccurrent tags. Sánchez developed another visualization algorithm, based on the Rule of Thirds in which there are 8 groups of tags and a central group that applies a radial 


\section{Distributed PERSONAL LEARNING ENVIRONMENTS: TOWARDS A SUITABLE ARCHITECTURE}

representation for the seven tags that have the highest cooccurrence frequency. Then 7 groups are filled with tags related to each one of the center tags. The eighth group is filled with tags with no co-occurrence with the center ones. It also allows pivot browsing, tag labeling to create semantic networks and tag definition (powered by Wikimedia projects) to make disambiguation easier. This method will allow one to represent 400 tags in a medium size screen, taking advantage of all the representation space (Figure 5).

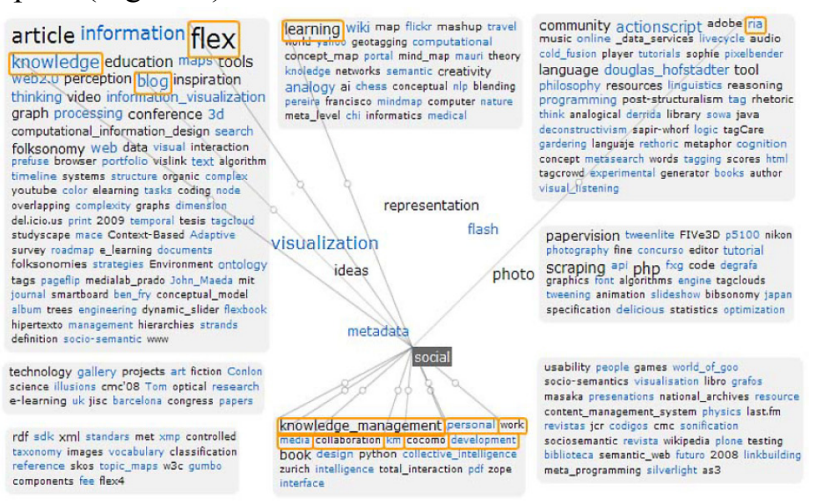

Figure 5. Advanced tag visualization [29].

\section{B. Structured Content}

\section{1) Concept}

Most learners are used to studying structured content, and most LMSs use this type of representation (Moodle [30], Sakai [31], Dokeos [32],...). All their books are structured in sections or units, each of which can have subsections and so on, creating a content tree or structure. It's easy to organize the study and estimate the time that each section will consume with just having a look at its subsections when using this model. If we have a big collection of unstructured resources, even if they are tagged and ordered by date, tag co occurrence or other property, it could be difficult to organize our study without a structure. So it seems that many students would use a social bookmarking tool that would allow them to organize their bookmarks and even create their own courses through structured bookmarks. These courses could also be shareable and users should be able to create them collaboratively.

A nice tool built specifically to be the main part of a PLE is Graasp [33]. It allows one to create spaces, which can contain other spaces, resources, contacts or widgets, creating a structure (see Figure 6). Graasp has the ability to enforce contextual aggregation of its four categories of entity: People, Spaces, Assets and Tools. To increase usability, almost all items are drag \& drop. It also uses recommendation to empower resource and people discovery, and allows element tagging. This tagging is not as advanced as it is in specific social bookmarking tools.

2) Visualization

The main visualizations for structured content are hierarchical structures (tree structures) and mind maps (see next section).

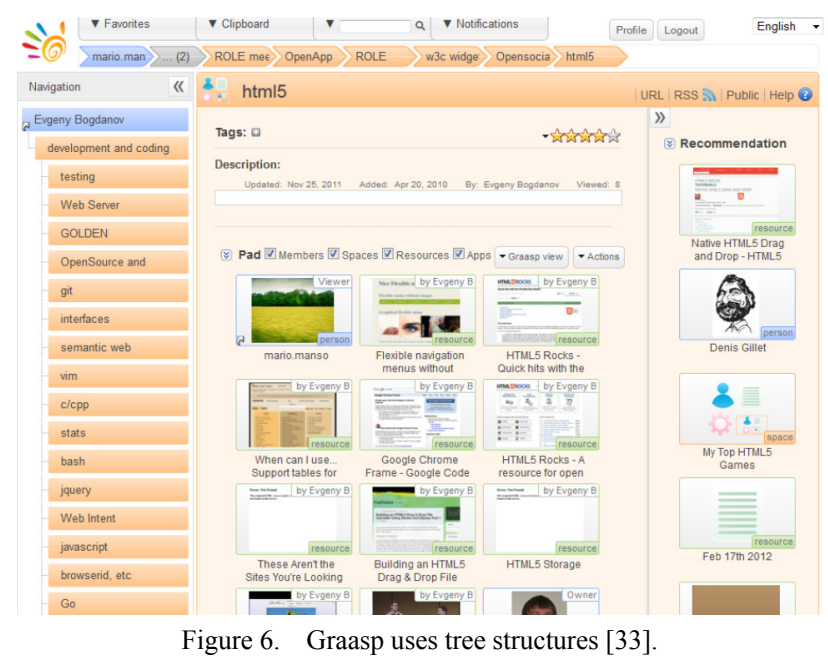

C. Concept Maps and Mind Maps

A Concept Map (CM) [34] is a graphical way of representing and organizing knowledge. It is comprised of nodes and links, arranged in some order to reflect the information of the domain being represented (see Figure 7). Nodes symbolize concepts, and links represent relationship between concepts; both concepts and links are labeled and may be categorized. It's been proved that CMs are a powerful instructional tool [34][35][36][37]. It can also be used as a course design developer.

$\mathrm{CMs}$ are meant to represent relationships among concepts, being a powerful tool to analyze from simple to very complex concepts. This capability to represent and organize knowledge makes CMs a great tool to be included in a PLE. The simpler case of use of a CM could be the representation of a structured course, with the added value of the information about the relationship among nodes. If we remove this relationship information, it becomes a mind map.

It has the following advantages:

- CMs force students to make valid connections among concepts, developing their conceptual understanding in opposition to rote learning.

- CMs Enables the students to have a global point of view of any studied subject.

- It provides a low tech vehicle that enables students to represent graphically their knowledge, and to share it with instructors and other students.

- It shifts the emphasis from inert, static knowledge to contextually-embedded knowledge; from isolated facts to theoretical frameworks of related concepts.

- It can be used as graphic organizers or course development tools.

It also has some disadvantages:

- In an institutional learning context, evaluation can become more time-consuming for instructors, especially in large classes. Comparisons among students are more difficult.

- Learners who have developed a strong facility for rote learning of verbal knowledge sometimes find CMs intimidating. 
- Building CMs maps is a demanding cognitive task that requires training.

There are a lot of tools to do this, also online like WiseMapping [38], Mind42 [39], MindMeister [40]... or desktop applications like CMapTools [41], FreeMind $[42], \ldots$

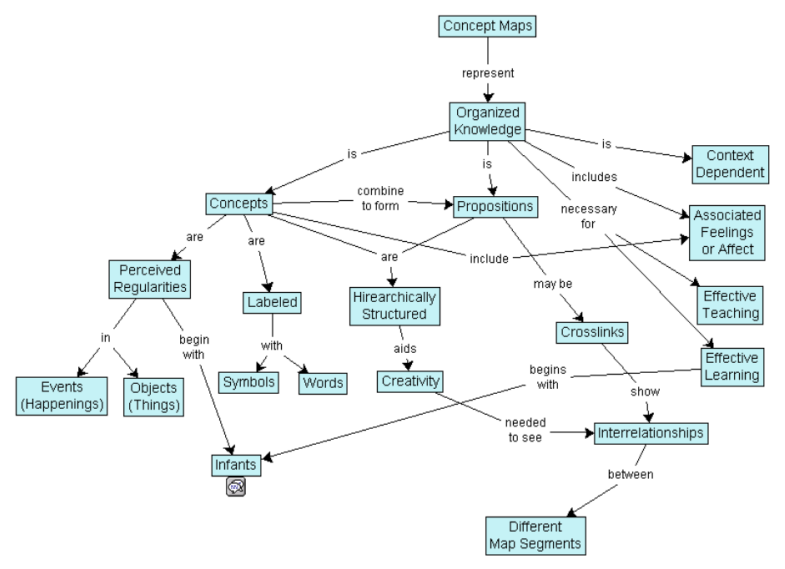

Figure 7. Example of a concept map.

\section{INTER-APPLICATION COMMUNICATION}

Most of the tools used to form a PLE are standalone applications, developed without the tool set idea in mind. Thus, they are not ready to communicate with other third party applications or to be integrated into an LMS or any larger system or heterogeneous tool set. The one and only communication link among all the applications of a multi-application PLE is the user. Even when using a mashup to integrate several applications, they are not connected to each other. The developers of the applications are not aware of this interoperability problem, as they see their applications as standalone.

It would be desirable to get all these different tools to act as a tool set, which could exchange data and be responsive to events happening in other applications. There are several alternatives to solve this interoperability problem. We selected the following ones:

\section{A. IMS LTI}

IMS LTI is a standard to allow remote tools to be integrated into a learning environment. The basic use case of this specification is to allow the seamless connection of web-based, externally hosted tools, to a learning environment. Eventually, users are provided with access to the remote tools as if they were available in the same server as the LMS. IMS LTI proposes a web protocol and an API to support this integration without having to develop and maintain custom integrations. In this way, IMS LTI is an important step ahead in the field of distributed e-learning systems, as long as it allows extending the functionalities of the LMS with the aid of external tools.

The LTI specification has been developed following a two-step effort. Initially, it published a reduced version of the specification named as Basic LTI [43]. This version was focused on the launch of the remote tools and on the provision of user access to such tools in a seamless and authenticated way. More recently, LTI version v1.1 was published [44] with the complete specification dealing with new issues, covering the outcomes transfer from the tools to the main system based on LIS Basic Outcomes. Now, version 2 introduces a new architecture for implementing LTI, being backwards compatible. It Implements a new model that specifies services using REST APIs in JSON format [45].

The LTI specification uses some specific terminology as it is represented in Figure 8. The learning environment is referred to as the "Tool Consumer" (TC) as it "consumes" the tool. The external tool is called the "Tool Provider" (TP) as it "provides" the tool that is going to be used from the "consumer".

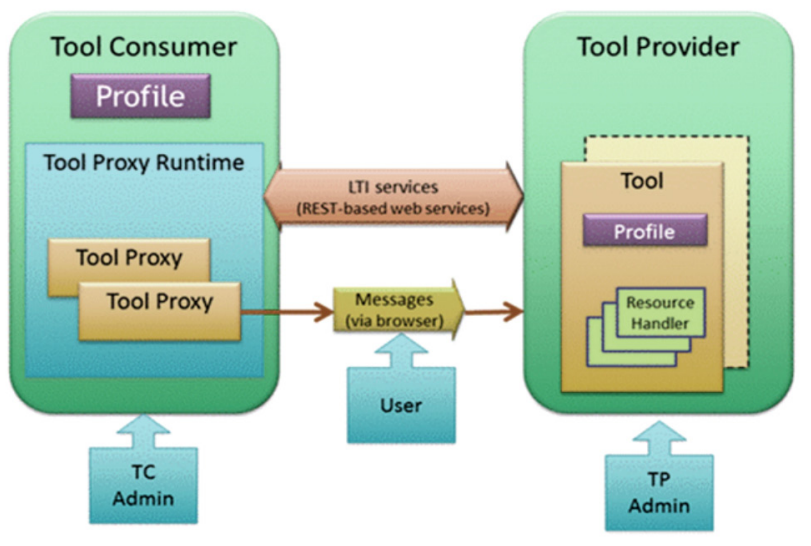

Figure 8. IMS LTI overview [45].

This option requires all the tools to be modified to adopt the LTI standard. IMS LTI is very simple and easy to implement as we know due to our experience in Game-Tel Project [46]. Anyway, LTI integration level is halfway between hard and soft integration and was been created to be in a centered architecture. It allows user authentication without OpenID (using OAuth protocol [47] with Single Sign On (SSO) and outcomes transfer, but it doesn't allow tool flow control.

\section{B. Tool Binding Adapter Middleware}

This middleware has been developed by Jorge Fontenla [48][49][50] to support the hard integration of tools into a LMS, allowing transparent access to external tools through SSO, exchanging data with the tool, subscribing to tool's events and adapting its workflow, following a SOA architecture. It does this using a Tool Binding Adapter Server (provides the LMS with a unified set of methods called Generic Tool Interface and adapts the calls to these methods into methods of the API of the tool, using information provided by the Binding Rules), Binding Rules (provides a relationship between the generic methods and the methods of the API of the tool), a Shared Vocabulary (categorizes all the possible terms and concepts concerning the inner structure and operation of the tool) (see Figure 10 and Figure 9).

With this middleware, hard integration can be achieved through the following steps:

- Creating a working account (i.e. an instance) for each user at the tool.

- Transferring from the LMS to the tool all data that the user may need in order to carry out his/her tasks. 

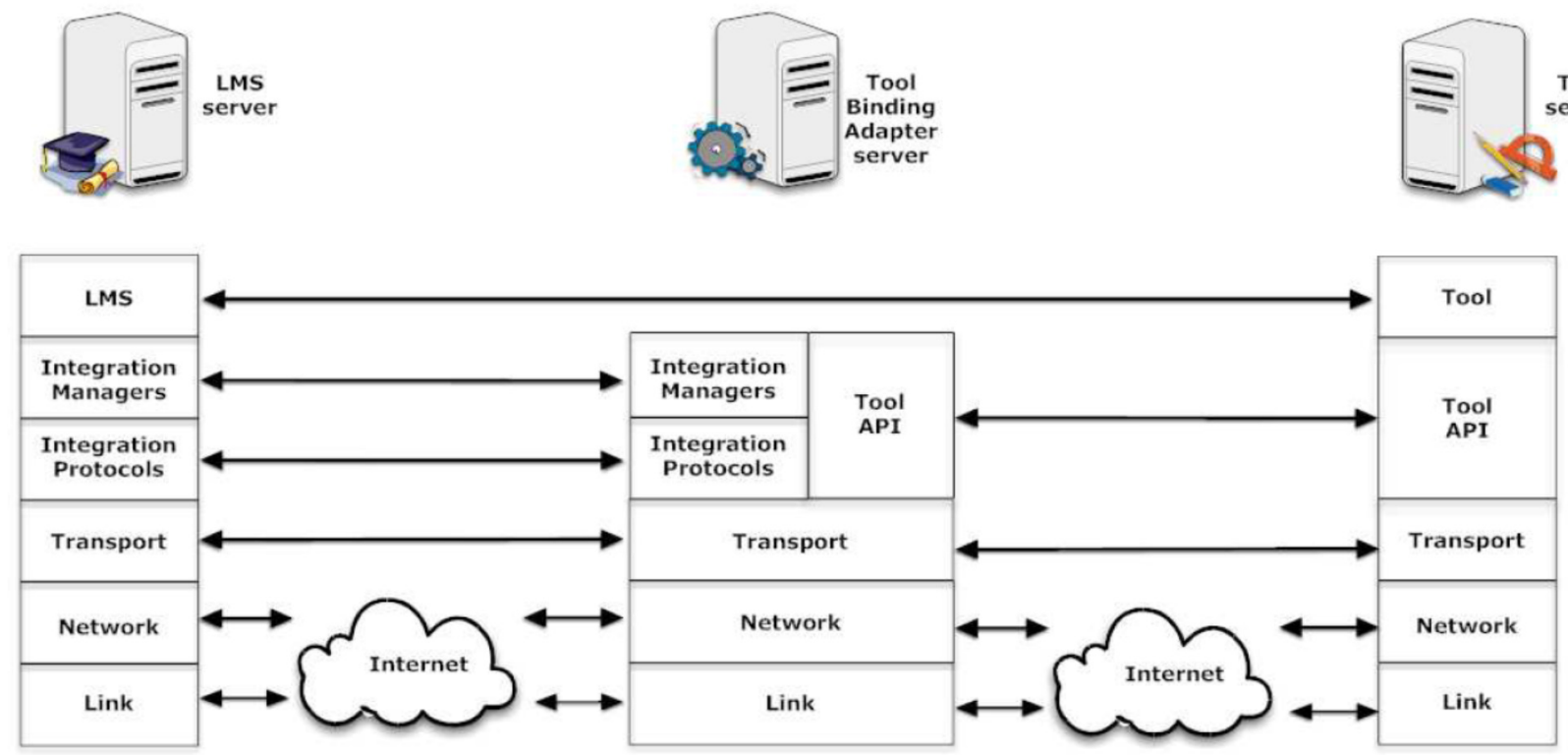

Figure 9. TBA Middleware protocol stack [50].

- Establishing some access permission over these data and some pieces of functionality of the tool.

- Subscribing to events resulting from the operation of the tool by its users.

- Authorize the users to access his/her instance or account.

- Alter the workflow of the tool according to the event data received.

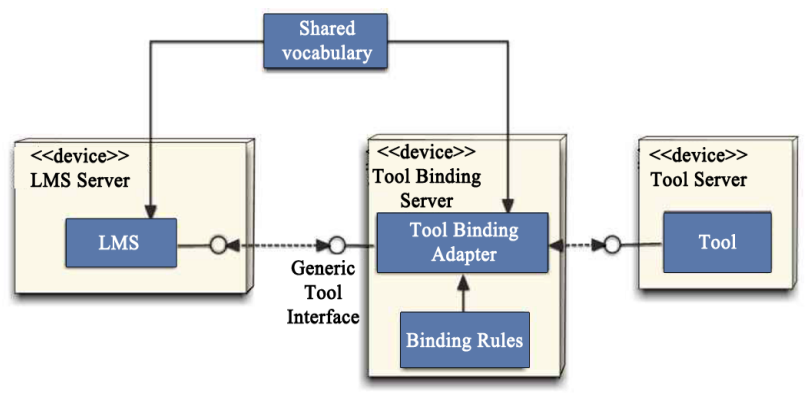

Figure 10. Component diagram of TBA Middleware [50].

This alternative goes a step ahead of other tool integration initiatives, providing a unique and well defined reference frame to integrate in a controlled way from Web2.0 applications to ad-hoc developed tools, but this may not be easy to implement. Using the API of the tool there is no need of modifying the tool itself. It may be necessary to create specific binding rules for every application, and the definition of a common vocabulary could be non-trivial. Our case is much simpler since we don't need to change the workflow of any application, but this could be a good starting point.

\section{Distributed PLE Case}

The two solutions presented before have been developed to integrate external tools into a LMS. Our use case is slightly different, since we have many different applications and none of them act as a "mother ship" or center application. It is important to consider that many of the developers won't modify their applications if the solution proposed doesn't become a renowned standard. The solution must be transparent to the user, but also needs to be easy to implement for the developers. It would be desirable not to make any app modification at all.

In order to make this possible, there are two possibilities:

1. Develop an inter-application communication protocol or standard, so it can be adopted by the developers of the implied applications.

2. Develop an inter-application communication middleware using an existing standard or a new one that act as a coordinator of a set of any configured applications. This would act as a communications center and common data storage. It is necessary to have a public API for the apps to be supported, so each application should be integrated separately.

\section{ARCHITECTURE}

\section{A. Requirements}

To achieve our goals, we define a simple set of requirements:

- Modifications to the applications should be minimal or none.

- It has to provide authorization management.

- Data must be updated among applications that manage the same information. For example, learners can use any of the three resource management tool types. If he/she uses more than one, data modifications must be updated in the others.

- Learners must be able to choose whether they want to synchronize content or not.

- A data format for each type of data exchange should be defined. A common vocabulary is needed. 


\section{B. Towards a Solution}

Using the cases stated in section V.C we have two options, as seen in Figure 11 and Figure 12.

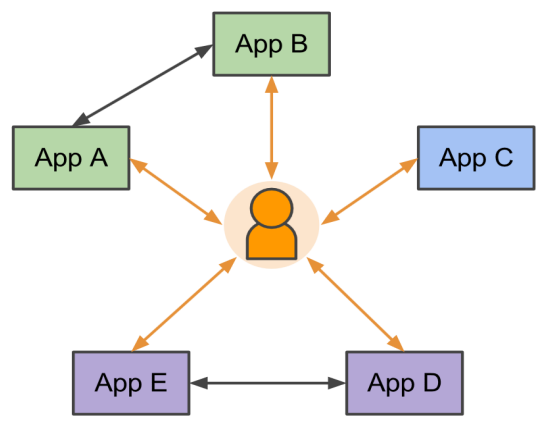

Figure 11. Case 1: inter-application communication protocol.

In case 1 we see that there is no architecture at all. An inter-application protocol is used and all these crossed communications are application dependent. At the same time, user must configure in each app which apps it should communicate to, and some options about what to communicate should be offered. All apps should have a listener to receive incoming data even if they are not in use at the moment. This option seems to be unfeasible since despite the definition of a standard protocol and data formats to support all these communications it needs the brands and developers acceptance and compromise to develop it. Commercial interests would be a barrier since many of the apps that have to communicate with each other are direct competitors.

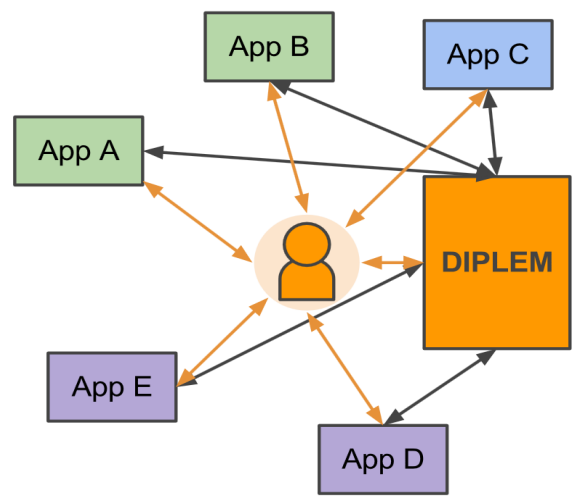

Figure 12. Case 2: Distributed PLE Manager.

On the other hand, case 2 is not application dependent. It can be a great solution to this communication and data exchange problem. We named it Distributed Personal Learning Environment Manager (DIPLEM). This solution is based on the Tool Binding Adapter Middleware, as it can be considered a particular and simple case of use of that powerful system. In this case there is no need of flow control (applications won't be allowed to control other application, their relationship is horizontal) and permissions control (this is app dependent). Granting authorization, access and data transfer is enough to make it work. Information can be synchronized by user request or periodically updated. DIPLEM interface has to offer the learner the possibility to synchronize the tools he/she is using to learn.

\section{Data Format}

Each application has its own data formats, variable names,... For example, a calendar tool could have a method called newcalendar(). The generic command for that would be createInstance (), so there is a need of defining a common vocabulary, or to configure each application individually in DIPLEM and associate the generic commands to the API's.

Below that, social bookmarking tools, structured content tools and concept map tools have different native formats. There is a need to establish a common format that can contain the information that these three types of tools need. Also, a conversion to each app format is needed, and has to be implemented into DIPLEM. A good starting point for that could be the Freemind's $\mathrm{mm}$ format, which is supported by most of the concept map and mind map tools [51]. The $\mathrm{mm}$ format is an XML text format consisting of tagged objects. This format can also be used to store the information of courses built in a structured content tool, if adapted. That conversion is simple and can be done into DIPLEM before sending the data to the other applications. Here is an example of this raw format:

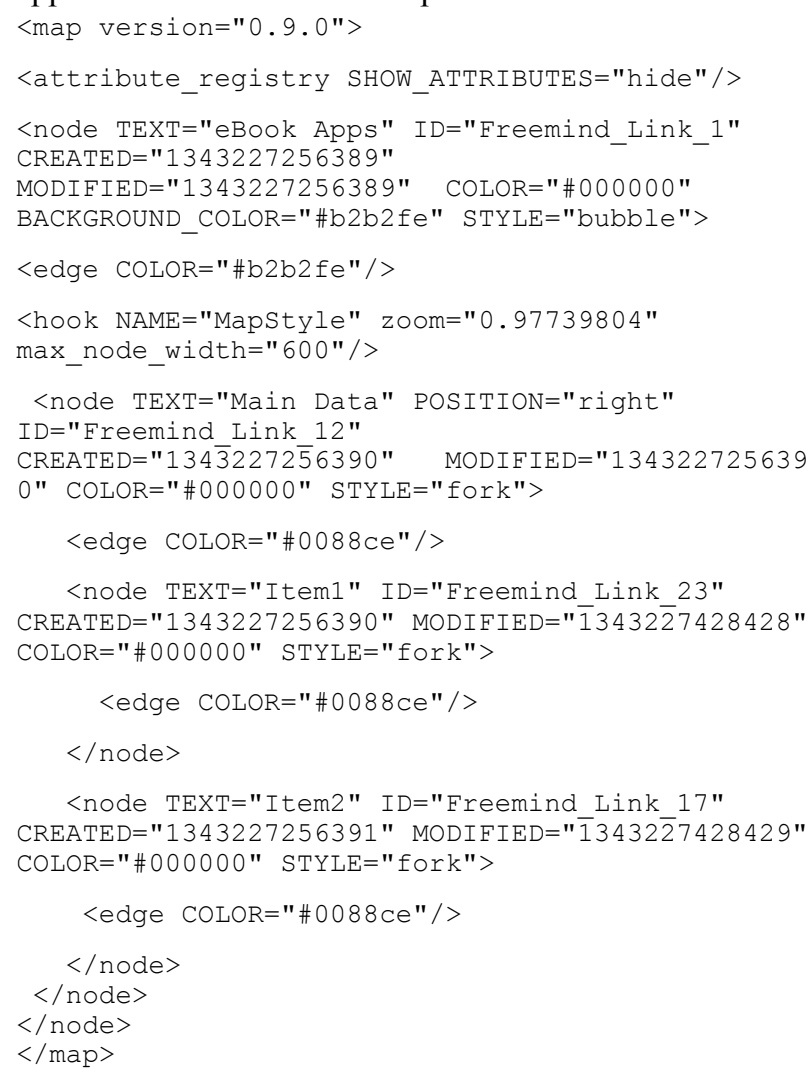

We are developing an extended $\mathrm{mm}$ format to include user tags (to be used by social tagging tools) and information about relationships between nodes (to be used in more complex CM tools like CMapTools). This way, every node can have a name or title, a set of tags, node relationships information and other node information (notes or description, html links, node appearance...). It has to be able to contain all the typical information used by any of the three types of information and resources management tools seen in section IV. 


\section{RELATED WORK}

Responsive Open Learning Environments (ROLE) is an European collaborative project with 16 internationally renowned research groups from 6 EU countries and China. ROLE technology is centered around the concept of SRL that creates responsible and thinking learners that are able to plan their learning process, search for the resources independently and learn and then reflect on their learning process and progress. ROLE's main objective is to support teachers in developing the open personal learning environments for their students where they can train each of the phases mentioned. ROLE has just entered the fourth project year, where their main endeavor is to test the already operating learning environment and widgets [52] (ROLE project website). ROLE Project are also the developers of Graasp, a powerful tool presented in section IV.B.

At this moment they are developing an extension of the W3C widget specification to include widget interoperability, end-user data mobility as a basis for manual widget orchestration, user behavior mining - for extracting behavioral patterns - as a basis for automated widget orchestration, and infrastructure [53]. This can be an important contribution to interoperability standards.

\section{CONCLUSIONS}

At present there are powerful tools to build a PLE or that could be a PLE themselves, even though they may not cover the needs of some learners. It's obvious that these tools need a minimum amount of users. Without enough users, recommendations, social tagging, social bookmarking and many collaborative features won't work properly.

We analyzed several PLE models and chose the distributed application model. We analyzed the main information management models, as they should be the main tool of a PLE and an essential tool for learning. We analyzed two communication methods (one of them is a standard) to solve the inter-application communication problem. Finally, we propose a possible solution to make a distributed application PLE responsive to itself, to make it work as a large tool and get as close as possible to the perfect PLE. This opens a new path for us to work on a non-invasive interoperability system which doesn't require application modifications to work.

\section{ACKNOWLEDGMENT}

Research supported by the Spanish Ministerio de Ciencia e Innovación under grant "Methodologies, Architectures and Standards for adaptive and accessible elearning (Adapt2Learn)', (TIN2010-21735-C02- 01), the European Regional Development Fund (ERDF) and the Galician Regional Government under project $\mathrm{CN}$ 2012/260 "Consolidation of Research Units: AtlantTIC".

\section{REFERENCES}

[1] Gillet, D., Law, E. L., \& Chatterjee, A. Personal Learning Environments in a Global Higher Engineering Education Web 2.0 Realm. EDUCON,Madrid,Spain: IEEE Xplore Electronic Library, pp. 897-906, 2010.

[2] van Harmelen, M. "Personal Learning Environments" in International Conference on Advanced Learning Technologies (ICALT). University of Manchester.
[3] Attwell, G., "The personal learning environments - The future of eLearning?" eLearning Papers, vol. 2(1), 2007.

[4] Pintrich, P. The role of motivation in promoting and sustaining self-regulated learning. International Journal of Educational Research, vol. 31, issue 6, pp. 459-470, 1999. http://dx.doi.org/10.1016/S0883-0355(99)00015-4

[5] Adell, J., Castañeda, L., "Los Entornos Personales de Aprendizaje (PLEs): una nueva manera de entender el aprendizaje," in Claves para la investigación en innovación y calidad educativas. La integración de las Tecnologías de la Información y la Comunicación y la Interculturalidad en las aulas. Alcoy: Marfil, 2010.

[6] Valtonen, T. et al., "Perspectives on personal learning environments held by vocational students", Computers \& $\begin{array}{llll}\text { Education, } & 58, & \text { pp. } & 732-739,\end{array}$ http://dx.doi.org/10.1016/j.compedu.2011.09.025

[7] Castañeda, L., Soto, J., "Patchworking Web 2.0 for learning: Introducing ICT as a professional tool building Personal learning Environments". Personal Learning Environment Conference (PLEC). Barcelona, 2010.

[8] Martín, R., Torres, R., "Ventajas pedagógicas en la aplicación del PLE en asignaturas de lengua y literatura de educación secundaria. Análisis de cinco experiencias", in Personal Learning Environment Conference (PLEC). Barcelona, 2010.

[9] Fiedler, S., \& Väljataga, T."Personal learning environments: concept or technology?" Personal Learning Environment Conference (PLEC). Barcelona, 2010.

[10] Milligan C.D et al., "Developing a Reference Model to Describe the Personal Learning Environment", in Innovative Approaches for Learning and Knowledge Sharing; First European Conference on Technology Enhanced Learning, EC-TEL, Crete, Greece, October 1-4, 2006 Proceedings, 2006, pp 506-511.

[11] Cetis, "PLEX consumes XCRI", Internet: http://www.elframework.org/projects/xcri/plex_consumes_xcri.gif /view.html, 2006 [May. 2013].

[12] Wang, F. et al., "Construct Personal Learning Environment Based on Web2.0" in Management and Service Science, Wuhan, IEEE, 2009, pp. 1-4.

[13] Soylu, A. et al., "Mashups by Orchestration", MEDES, San Francisco, USA, ACM,2011, pp. 226-234.

[14] PLEF Team, "Personal Learning Environment Framework", Internet: $\quad$ http://eiche.informatik.rwthaachen.de:3333/PLEF/index.jsp, 2008 [May 2013].

[15] SlideShare Inc, "Slideshare", Internet: http://www.slideshare.net/, 2013 [May 2012].

[16] Youtube, "YouTube", Internet: http://www.youtube.com/, 2013 [May 2013].

[17] OpenId, "OpenId website”, Internet: http://openid.net/, 2013 [May 2013].

[18] Elgg Foundation, Curverider Ltd., "Elgg, Open Source Social Networking Engine". Internet: http://elgg.org, 2013 [May 2013].

[19] Nevibes. "Netvibes.com". Internet: http://www.netvibes.com, 2013 [May 2013].

[20] Ning. "Ning, social network creation patform". Internet: http://www.ning.com/, 2013 [May 2013]

[21] Popurls, "Popurls, genuine aggregator", Internet: http://popurls.com/, 2013, [May 2013]

[22] Pageflakes, "PageFlakes", Internet: http://www.pageflakes.com/, 2013 [May 2013]

[23] Henri, F., Charlier, B.“Personal learning environment: A concept, an application, or a selfdesigned instrument?" Information Technology Based Higher Education and Training (ITHET), 2010, pp. 44-51. Cappadocia: IEEE Electronic Library.

[24] Sclater, N. "Web 2.0, Personal Learning Environments, and the Future of Learning Management Systems". ECAR, Research Bulletin, 2008. Boulder, Colorado, USA.

[25] AVOS Systems, "Delicious", Internet: http://delicious.com/, 2013 [May 2013]

[26] Diigo Inc, "Diigo: Collect and Highlight", Internet: http://www.diigo.com/, 2013 [May 2013] 


\section{SPECIAL FOCUS PAPERS \\ Distributed PERSONAl LEARNING ENVIRONMENTS: TOWARdS A SUITABLE ARCHITECTURE}

[27] Vander Wal, T., "Folksonomy Coinage and Definition", Off of the Top (blog), Internet: http://vanderwal.net/folksonomy.html, 2007 [May 2013].

[28] J. Trant, "Studying Social Tagging and Folksonomy: A Review and Framework".Journal of Digital Information. Special Issue on Digital Libraries and User Generated Content. 2008.

[29] Sánchez, F., Llamas, M., "Visualizing tags as a Network of relatedness", Frontiers in Education Conference (FIE), 2009, pp. W2A.1-W2A.6.

[30] Moodle."Moodle website. Moodle Trust". Internet: http://moodle.org/, 2013 [May 2013].

[31] Sakai Foundation. "Sakai". Internet: http://www.sakaiproject.org/, 2013 [May 2013]

[32] Dokeos. "Dokeos". Internet: http://www.dokeos.com/, 2013 [May 2013]

[33] Graasp. “Graasp”, Internet: http://graasp.epfl.ch/, 2013 [May 2013]

[34] Rueda, U. et al., "Evaluating a Concept Map Editor with nontechnical students", International Conference on Advanced Learning Technologies (ICALT), 2008, pp. 405-407.

[35] Taber, K.S."Student reaction on being introduced to concept mapping". Physics Education, vol. 29(5), pp. 276-281, 1994. http://dx.doi.org/10.1088/0031-9120/29/5/003

[36] Brandt, L. et al., "The impact of concept mapping and visualization on the learning of secondary school chemistry students". International Journal of Science Education, vol. 23(12), 2001, pp. 1303-1313. http://dx.doi.org/10.1080/095006901100 $\underline{49088}$

[37] Stoddart, T. et al. "Concept maps as assessment in science inquiry learning - a report methodology". International Journal of Science Education, vol. 22(12), 2002, pp. 1221-1246. http://dx.doi.org/10.1080/095006900750036235

[38] Wisemapping, "Wisemapping - Visual Thinking Evolution", Internet: http://www.wisemapping.com/, 2013 [May 2013]

[39] IRIAN Solutions GmbH, “Mind42”, Internet: http://mind42.com/, 2013 [May 2013]

[40] MeisterLabs, "MindMeister", Internet: http://www.mindmeister.com/es, 2013 [May 2013]

[41] IHMC, "CmapTools", Internet: http://cmap.ihmc.us/, 2013 [May 2013]

[42] Freemind, "Freemind Wiki", Internet: http://freemind.sourceforge.net/wiki/index.php/Main_Page, 2013 [May 2013]

[43] McFall, G. and Neumann, L. "IMS learning tools interoperability basic LTI implementation guide v1.0 Final", Internet: http://www.imsglobal.org/lti/ 2010 [May 2013]

[44] McFall, G. and Neumann, L. "IMS GLC learning tools interoperability implementation guide v1.1 Final", Internet: http://www.imsglobal.org/lti/v1p1pd/ltiIMGv1p1 2012[May 2013]

[45] McFall, G. et al., "IMS GLC learning tools interoperability implementation guide v2.0 Public Draft" Internet: http://www.imsglobal.org/lti/v2p0pd/ltiIMGv2p0pd.html 2012 [May 2013]
[46] Caeiro, M. et al., "Design of Flexible and Open Learning Management Systems using IMS Specifications. The Game-Tel Experience". Journal of Research and Practice in Information Technology (JRPIT), Vol. 44, No. 2. 2012

[47] Oauth, "OAuth protocol, Internet Engineering Task Force". Internet: http://oauth.net/ 2013 [May 2013]

[48] Fontenla, J. et al., "A SOA Architecture to Improve the Tailorability and Extensibility of e-Learning Systems", IEEE Latin America Transactions, vol. 8 (2), April 2010. http://dx.doi.org/10.1109/TLA.2010.5514439

[49] Fontenla, J., et al., "A Middleware for the Integration of Thirdparty Learning Tools in SOA-based Learning Management Systems", EDUCON 2010, pp. 867-875.

[50] Fontenla, J. "Contributions to the integration of external tools in eLearning systems", PhD Thesis, University of Vigo, Spain, 2010.

[51] Freemind, "Freemind format supported apps", Internet: http://en.wikipedia.org/wiki/List of concept- and mindmapping_software, 2013, [May 2013]

[52] ROLE, "ROLE project website", Internet: http://www.roleproject.eu/ 2013 [May 2013]

[53] Soylu, A. et al., "Mashups and Widget Orchestration", in The International Conference on Management of Emergent Digital EcoSystems (MEDES), San Francisco, California, 2011, ACM, pp. 226-234.

\section{AUTHORS}

Mario Manso-Vázquez received the MSc degree in telecommunication engineering in 2007 at the University of Vigo, Spain. Nowadays he is a PhD student, and is also working as a software engineer and graphic designer at the Department of Telematic Engineering at the same university. He has worked as a software engineer and graphic designer and participated in Game-Tel e-learning project as software developer and interoperability manager. (e-mail: mario.manso@uvigo.es).

Dr. Martín Llamas-Nistal, received his Engineering degree (1986) and his PhD degree (1994) from the Polytechnic University of Madrid. He is currently an associate professor at the Department of Telematics Engineering at University of Vigo. He is author or coauthor of more than 200 papers in peer-reviewed international refereed journals and conference proceedings. He has directed several national and international research projects in telematics and technology enhanced learning fields. (e-mail: martin@uvigo.es).

This article is an extended and modified version of a paper presented at the EDUCON2013 conference held at Technische Universität Berlin, Berlin, Germany from March 13-15, 2013. Submitted 13 May, 2013. Published as re-submitted by the authors on 16 May 2013. 